\title{
Country of Origin Narratives of Brand Image for Japanese Cosmetics Through a Consumer Lens: An Abstract
}

\author{
Stephanie Slater and Maki Umemura
}

\begin{abstract}
Japanese companies such as Toyota, Suzuki, Toshiba and Sony are internationally known for the brands that they have built in the manufacturing and electronics industries. But, interestingly, these successes have not been reproduced in sectors such as cosmetics, where Japanese brands have struggled to achieve the same global awareness and recognition as products from the USA and France. Instead, what has materialised is a scenario where Japanese cosmetic brands are relatively unknown outside Asia. This raises interesting questions about the way brands and products gain recognition between and across markets. Through a series of focus groups, we explore why Japanese cosmetic products are largely unknown outside Japan, given the country's success in other sectors, such as automobiles, which have strong brand presence and awareness. We evaluate the reasons behind this lack of awareness by examining if consumer preferences towards particular cosmetic brands are linked to antecedents such as geography, country-of-origin effect and national stereotypes. The research unravels the antecedents that may have given rise to asymmetric performance in the sector by analysing cosmetic brand knowledge, understanding and preferences through appropriate consumer lenses. In this paper, we discuss consumer perspectives of beauty and explain why and how culture, identity and geography can lead to uncertainties and different perspectives of what beauty means when positioning products in different settings. We show how these factors inform attitudes towards products and consumption. Our initial findings show why understanding this phenomenon is important in understanding globalisation barriers and outlining the implications for cosmetic companies with global ambitions.
\end{abstract}

\section{References Available Upon Request}

Acknowledgements Funding body acknowledgement: This project is funded by the Great Britain Sasakawa Foundation. The authors are grateful to the foundation for both their support and the funding of this project. We also express our heartfelt thanks to colleagues and friends in Japan, the UK and France for the help that they gave in setting up this project.

\author{
S. Slater $(\varangle) \bullet$ M. Umemura \\ Cardiff University, Cardiff, UK \\ e-mail: SlaterS@cf.ac.uk; UmemuraM@cardiff.ac.uk
}

